\title{
ON PROPERTIES OF GROUP CLOSURES OF ONE-TO-ONE TRANSFORMATIONS
}

\author{
INESSA LEVI \\ (Received 4 January 2002; revised 3 November 2004) \\ Communicated by D. Easdown
}

\begin{abstract}
For a permutation group $H$ on an infinite set $X$ and a transformation $f$ of $X$, let $\langle f: H\rangle=\left\langle\left\{h f h^{-1}\right.\right.$ : $h \in H\})$ be a group closure of $f$. We find necessary and sufficient conditions for distinct normal subgroups of the symmetric group on $X$ and a one-to-one transformation $f$ of $X$ to generate distinct group closures of $f$. Amongst these group closures we characterize those that are left simple, left cancellative, idempotent-free semigroups, whose congruence lattice forms a chain and whose congruences are preserved under automorphisms.
\end{abstract}

2000 Mathematics subject classification: primary $20 \mathrm{M} 20$.

Keywords and phrases: semigroup, transformation, permutation group, conjugates, centralizer, congruence, congruence lattice, left simple, automorphism group, inner automorphism.

\section{Introduction}

Let $X$ be a nonempty set, and let $H$ be a subgroup of the symmetric group $\mathscr{G}_{X}$ on $X$. Denote the alternating subgroup of $\mathscr{G}_{X}$ by $\mathscr{A}_{X}$. Given a transformation $f$ of $X$, the semigroup

$$
\langle f: H\rangle:=\left\langle\left\{h f h^{-1}: h \in H\right\}\right\rangle
$$

is the $H$-closure of $f$ (or a group-closure). For any semigroup $S$ of transformations of $X$, let $G_{S}=\left\{h \in \mathscr{G}_{X}: h S h^{-1} \subseteq S\right\}$ and say that $S$ is $H$-normal if $G_{S}=H$.

If $X$ is finite, then for a transformation $f$ of $X$ we have that $\left\langle f: \mathscr{A}_{X}\right\rangle$ and $\left\langle f: \mathscr{G}_{X}\right\rangle$ differ if and only if $f$ is a partial one-to-one nilpotent having the union of its image and domain equal to $X([5,8,7])$. In particular, if $f$ is a total transformation, then $\left\langle f: \mathscr{A}_{X}\right\rangle=\left\langle f: \mathscr{G}_{X}\right\rangle$, and the semigroup $\left\langle f: \mathscr{A}_{X}\right\rangle$ is a $\mathscr{G}_{X}$-normal semigroup. The papers cited above address the following problem for a finite set $X$ :

(c) 2005 Australian Mathematical Society 1446-7887/05 $\$ \mathrm{~A} 2.00+0.00$ 
PROBlem 1. Let $H_{1}, H_{2}$ be subgroups of $\mathscr{G}_{X}$, and let $f$ be a transformation of $X$. Find necessary and sufficient conditions for equality of the group closures $\left\langle f: H_{1}\right\rangle$ and $\left\langle f: H_{2}\right\rangle$.

The case of an infinite set $X$ is much more complex than that of a finite $X$. Here Problem 1 is addressed in the particular case when $X$ is infinite and the subgroups of $\mathscr{G}_{X}$ are normal. We establish necessary and sufficient conditions for two distinct normal subgroups $H_{1}$ and $H_{2}$ of $\mathscr{G}_{X}$ to produce equal group closures $\left\langle f: H_{1}\right\rangle$ and ( $f: H_{2}$ ) of a one-to-one total transformation $f$ of $X$.

A semigroup $S$ is said to be a $\Delta$-semigroup if its congruence lattice $\operatorname{Con}(S)$ forms a chain. A congruence $\theta$ on a semigroup $S$ is said to be invariant under an automorphism $\psi$ of $S$ if whenever $(f, g) \in \theta$ we also have $(\psi(f), \psi(g)) \in \theta$. For a transformation $f$ of $X$, let the defect of $f$ be the cardinality of the complement of its image $\operatorname{im}(f)$,

$$
\operatorname{def}(f)=|X-\operatorname{im}(f)| .
$$

The subset of all the points of $X$ shifted by $f$ is $S(f)=\{x \in X: f(x) \neq x\}$ and

$$
\operatorname{shift}(f)=|S(f)|, \quad \operatorname{fix}(f)=|X-S(f)| .
$$

Given an infinite cardinal $\gamma \leq|X|$, the Baer-Levi semigroup $B L(|X|, \gamma)$ is the semigroup of all total one-to-one transformations of $X$ with defect $\gamma$ [1]. Baer-Levi semigroups are idempotent-free left simple, left cancellative $\Delta$-semigroups whose congruences are preserved under automorphisms $([9,10,11])$. The following problem was raised in [10].

PROBLEM 2. Characterize all idempotent-free left simple, left cancellative $\Delta$-semigroups whose congruences are preserved under automorphisms.

Let $f$ be a total one-to-one transformation of $X$ such that $\gamma=\operatorname{def}(f)$ is infinite. If $\operatorname{shift}(f)=|X|$, then $\left\langle f: \mathscr{G}_{X}\right\rangle$ is a Baer-Levi semigroup $B L(|X|, \gamma)$. It was shown in [10] that for any $g \in B L(|X|, \gamma)$, the semigroup $\left\langle g: \mathscr{G}_{X}\right\rangle$ satisfies the conditions stated in Problem 2. In this paper, for a normal subgroup $H$ of $\mathscr{G}_{X}$, we characterize the $H$-closures of $f$ that satisfy the conditions stated in Problem 2, thereby generalizing the result of [10] and providing a contribution towards a solution of Problem 2.

\section{Background and main results}

Let $\mathscr{W}_{X}$ denote the semigroup of all the total one-to-one transformations of an infinite set $X$. For any infinite cardinal $\alpha$ not exceeding the cardinal successor $|X|^{+}$ of $|X|$, and any cardinal $\gamma \leq|X|$, let

$$
S(X, \alpha, \gamma)=\left\{f \in \mathscr{W}_{X}: \operatorname{shift}(f)<\alpha, \operatorname{def}(f)=\gamma\right\} .
$$


If $\aleph_{o} \leq \gamma<\alpha$, then $S(X, \alpha, \gamma)$ is a $\mathscr{G}_{X}$-normal subsemigroup of $\mathscr{W}_{X}$. If $\gamma=0$, then $S(X, \alpha, \gamma)$ is a normal subgroup of the symmetric group $\mathscr{G}_{X}$, and these groups together with the alternating group $\mathscr{A}_{X}$ constitute the set of all the non-trivial normal subgroups of $\mathscr{G}_{X}([12])$. For brevity, we write $S(X, \alpha, 0)$ as $S(X, \alpha)$.

\subsection{Decomposition of one-to-one transformations The elements of $\mathscr{W}_{X}-\mathscr{G}_{X}$} are referred to as non-permutations in $\mathscr{W}_{X}$. Just as any permutation may be written as a formal product of disjoint finite and infinite cycles, any non-permutation in $\mathscr{W}_{X}$ may be written (essentially uniquely) as a formal product of disjoint cycles (finite or infinite) and chains [3] (see Proposition 2.1 below). As usual, transformations $f$ and $g$ are disjoint if $S(f) \cap S(g)=\emptyset$. Let $A$ be a set of pairwise disjoint transformations of $X$. The formal product of $A$ is a transformation of $X$, denoted by $\prod\{f: f \in A\}$, defined by the following formula:

$$
\prod\{f: f \in A\}(x)= \begin{cases}f(x), & \text { if } f \in A \text { and } x \in S(f) ; \\ x, & \text { if } x \in X-\cup\{S(f): f \in A\},\end{cases}
$$

where $x \in X$. If $A \subseteq \mathscr{W}_{X}$ then $\prod\{f: f \in A\}$ is also in $\mathscr{W}_{X}$. For a countable ordered subset $Y=\left\{y_{1}, y_{2}, y_{3}, \ldots\right\}$ of $X$, let $\left[y_{1} y_{2} y_{3} \ldots\right)$ denote the transformation $f \in \mathscr{W}_{X}$ such that $f\left(y_{i}\right)=y_{i+1}$ for $i=1,2,3, \ldots$, and $f(x)=x$ for all $x \in X-Y$. The transformation $f=\left[y_{1} y_{2} y_{3} \cdots\right)$ is called a chain. Note that $X-\operatorname{im}(f)=\left\{y_{1}\right\}$ and $\operatorname{def}(f)=1$. The following result has been proved in [3]:

PROPOSITION 2.1. Let $f$ be a non-identity transformation in $\mathscr{W}_{X}$. Then $f$ is a formal product of pairwise disjoint cycles and chains, $f=\prod\{g: g \in A\}$, with no $g \in A$ being a 1-cycle. The number of chains in $A$ is equal to $\operatorname{def}(f)$. If $f=\prod\left\{g: g \in A^{\prime}\right\}$ is another such product then $A=A^{\prime}$.

We refer to the form $\prod\{f: f \in A\}$ of $f$ as the cyclic-chain decomposition of $f$. Let $\mathscr{C} h_{X} \subseteq \mathscr{W}_{X}$ be the set of all formal products of disjoint chains. Proposition 2.1 assures that every transformation $f \in \mathscr{W}_{X}$ can be written as a product of two unique disjoint transformations $f_{p} \in \mathscr{G}_{X}$ and $f_{c} \in \mathscr{C} h_{X}$ (the subscripts $p$ and $c$ stand for permutation and chain correspondingly). We let $\varphi_{n}(f)$ denote the set of all the $n$-cycles in the cyclic decomposition of $f$, where $n$ is either a positive integer or $\infty$.

2.2. Main results The main results of this paper are stated in Theorems $2.2-2.4$ below. The remainder of the paper is concerned with the proof of these results.

THEOREM 2.2. Let $f$ be a non-identity transformation in $\mathscr{W}_{X}$, and let $H$ be a normal subgroup of $\mathscr{G}_{X}$. Then $\langle f: H\rangle$ is left simple if and only if

(1) $\operatorname{def}(f)=\gamma$ is infinite, 
(2) $H=S(X, \alpha)$ for some $\alpha>\gamma$,

(3) there exists a positive integer $m$ such that $\operatorname{shift}\left(f^{m}\right)<\alpha$.

Lemma 3.10 presents conditions in terms of the cyclic-chain decomposition of $f$ that are equivalent to Theorem 2.2 (3).

THEOREM 2.3. Let $f$ be a non-permutation in $\mathscr{W}_{X}$, and let $H$ be a normal subgroup of $\mathscr{G}_{X}$. Then $\langle f: H\rangle$ is a left cancellative, idempotent-free semigroup. If $\langle f: H\rangle$ is left simple, then it is a $\Delta$-semigroup whose congruences are preserved under automorphisms.

THEOREM 2.4. Let $\aleph_{o} \leq \alpha \leq \beta \leq|X|^{+}$and let $f$ be a non-identity transformation in $\mathscr{W}_{X}$.

(1) If $\alpha<\beta$, then $\langle f: S(X, \alpha)\rangle=\langle f: S(X, \beta)\rangle$ if and only if $\operatorname{shift}(f)<\alpha$.

(2) $\left\langle f: \mathscr{A}_{X}\right\rangle=\langle f: S(X, \beta)\rangle$ if and only if either

(a) $f$ is a finite permutation, or

(b) $\beta=\aleph_{o}$ and for some integer $k \geq 1$ either $\left|\varphi_{2 k}(f)\right| \geq 1$ or $\left|\varphi_{2 k-1}(f)\right| \geq 2$.

2.3. Properties of one-to-one transformations The first three statements of the following lemma are easily derived from elementary properties of one-to-one transformations, while the fourth statement presents a well-known result for permutations (see, for example, [12]), and has been proved for non-permutations in $\mathscr{W}_{X}$ in [3].

LEMMA 2.5. Let $f, g \in \mathscr{W}_{X}$. Then the following hold:

(1) $\operatorname{def}(f g)=\operatorname{def}(f)+\operatorname{def}(g)$.

(2) $S\left(h g h^{-1}\right)=h(S(g))$ for all $h \in \mathscr{G}_{X}$.

(3) If $g \notin \mathscr{G}_{X}$, then $\operatorname{shift}(g)$ is infinite and $\operatorname{shift}(f g) \leq \operatorname{shift}(f)+\operatorname{shift}(g)$, equality holding when $\operatorname{shift}(f) \neq \operatorname{shift}(g)$.

(4) $f=h g h^{-1}$ for some $h \in \mathscr{G}_{X}$ if and only if $\operatorname{def}(f)=\operatorname{def}(g)$, and $\left|\varphi_{n}(f)\right|=$ $\left|\varphi_{n}(g)\right|$ for all $n=1,2, \ldots, \infty$.

For transformations $f, g \in \mathscr{W}_{X}$, let $D(f, g)=\{x \in X: f(x) \neq g(x)\}$.

LEMMA 2.6. For $f, g, t \in \mathscr{W}_{X}$, if $t f=g$ then

$$
|D(f, g)| \leq \operatorname{shift}(t) \leq|D(f, g)|+\operatorname{def}(f) .
$$

ProOF. If $t f=g$, then $f(D(f, g)) \subseteq S(t) \subseteq f(D(f, g)) \cup(X-\operatorname{im}(f))$.

For a transformation $f$ of $X$, let $C_{\mathscr{G}_{X}}(f)=\left\{h \in \mathscr{G}_{X}: h f=f h\right\}$ denote the centralizer of $f$ in $\mathscr{G}_{X}$. The next two results, proved in [6], describe centralizers of transformations in $\mathscr{W}_{X}$. 
PROPOSITION 2.7. Take $f \in \mathscr{W}_{X}$ and write it as a product of disjoint transformations $f=f_{p} f_{c}$, where $f_{p} \in \mathscr{G}_{X}, f_{c} \in \mathscr{C}_{h_{X}}$. A permutation $h \in C_{\mathscr{G}_{X}}(f)$ if and only if $h \in C_{\boldsymbol{G}_{x}}\left(f_{p}\right), h\left(S\left(f_{c}\right)\right)=S\left(f_{c}\right)$, and for each chain $\left[x_{1} x_{2} x_{3} \cdots\right)$ in $f_{c}$, $\left[h\left(x_{1}\right) h\left(x_{2}\right) h\left(x_{3}\right) \cdots\right)$ is also a chain in $f_{c}$.

LeMMA 2.8. Let $g \in \mathscr{G}_{X}$. Then $C_{\mathscr{G}_{X}}(g) \leq \mathscr{A}_{X}$ if and only if $\mathrm{fix}(g) \leq 1$ and $g$ is a product of disjoint finite cycles of distinct odd lengths.

Let $\aleph_{o} \leq \alpha<\beta \leq|X|^{+}$and let $S(X, \alpha)$ and $S(X, \beta)$ be two distinct normal subgroups of $\mathscr{G}_{X}$, so $S(X, \alpha)<S(X, \beta)$. Lemma 2.10 gives a sufficient condition for equality of $\langle f: S(X, \alpha)\rangle$ and $\langle f: S(X, \beta)\rangle$ for $f \in \mathscr{W}_{X}$. The lemma assures equality of these group closures whenever $f$ shifts fewer than $\alpha$ elements of $X$ by demonstrating that both are just the minimal $\mathscr{G}_{X}$-normal semigroups containing $f$. These semigroups were described in [3], where the following has been proved.

Proposition 2.9. Let $f \in \mathscr{W}_{X}$ with $\operatorname{shift}(f)=\sigma$ and $\operatorname{def}(f)=\gamma$.

(1) If $\gamma \geq \aleph_{o}$, then $\left\langle f: \mathscr{G}_{X}\right\rangle=S\left(X, \sigma^{+}, \gamma\right)$.

(2) If $\gamma$ is a positive integer, then

$$
\left\langle f: \mathscr{G}_{X}\right\rangle \supseteq\left\{g \in \mathscr{W}_{X}: \operatorname{shift}(g) \leq \sigma, \operatorname{def}(g)=k \gamma, \text { for all integers } k \geq 4\right\} .
$$

Properties of $\mathscr{G}_{X}$-normal semigroups have been studied in a number of publications, see, for example, $[9,2,3,11,13,14]$.

LEMMA 2.10. Let $f \in \mathscr{W}_{X}$ with $\operatorname{shift}(f)=\sigma$ and let $\alpha$ be an infinite cardinal greater than $\sigma$. Then $\langle f: S(X, \alpha)\rangle=\left\langle f: \mathscr{G}_{X}\right\rangle$.

Proof. We only need to prove that $h f h^{-1} \in\langle f: S(X, \alpha)\rangle$ for any $h \in \mathscr{G}_{X}$. Let $Y=S(f) \cup S\left(h f h^{-1}\right)=S(f) \cup h(S(f))$. Then $|Y| \leq 2 \sigma<\alpha$, and the restrictions $f_{\mid Y}, h f h_{\mid Y}^{-1}$ are total transformations of $Y$. Also, $\operatorname{def}(f)=\operatorname{def}\left(h f h^{-1}\right)$ and $\left|\varphi_{n}(f)\right|=$ $\left|\varphi_{n}\left(h f h^{-1}\right)\right|$ for all $n=1, \ldots, \infty$ (by Lemma $2.5(4)$ ), so $\left|Y-\operatorname{im}\left(h f h_{\mid Y}^{-1}\right)\right|=$ $\left|Y-\operatorname{im}\left(f_{\mid Y}\right)\right|=\operatorname{def}(f)$ and $\left|\varphi_{n}\left(f_{\mid Y}\right)\right|=\left|\varphi_{n}\left(h f h_{\mid Y}^{-1}\right)\right|$ for all $n=1, \ldots, \infty$. Therefore, by Lemma 2.5 (4) again, there is a permutation $g \in \mathscr{G}_{Y}$ such that $h f h_{\mid Y}^{-1}=g f_{\mid Y} g^{-1}$. Define a permutation $\tilde{h} \in \mathscr{G}_{X}$ such that $\tilde{h}(x)=g(x)$, if $x \in Y$, and $\tilde{h}(x)=x$, if $x \in X-Y$. Then $\tilde{h} \in S(X, \alpha)$ and $h f h^{-1}=\tilde{h} f \tilde{h}^{-1} \in\langle f: S(X, \alpha)\rangle$.

\section{On algebraic properties of group closures}

Throughout suppose that $\alpha$ is an infinite cardinal. Let $f$ be a non-permutation in $\mathscr{W}_{X}$, and let $H$ be a normal subgroup of $\mathscr{G}_{X}$. Since the semigroup $\langle f: H\rangle$ consists of oneto-one transformations, it is left cancellative. Since any idempotent transformation of $X$ is the identity on its image, the semigroup $\langle f: H\rangle$ is idempotent-free. 
3.1. Left simplicity of $\langle\boldsymbol{f}: \boldsymbol{H}\rangle$ The following result shows that for any $f \in \mathscr{W}_{X}$, $\langle f: S(X, \alpha)\rangle$ is a subsemigroup of $S(X, \alpha)\langle f\rangle$ where $\langle f\rangle$ is the subsemigroup generated by $f$.

LEMMA 3.1. Let $f \in \mathscr{W}_{X}$ and $g=h_{1} f h_{1}^{-1} h_{2} f h_{2}{ }^{-1} \cdots h_{k} f h_{k}{ }^{-1}$ for some $h_{i} \in$ $S(X, \alpha), i=1,2, \ldots, k$. Then there exists $h \in S(X, \alpha)$ such that $g=h f^{k}$, and $|\operatorname{im}(g)-\operatorname{im}(f)|<\alpha$.

Proof. It suffices to show that given any $p \in S(X, \alpha)$ there exists $q \in S(X, \alpha)$ such that $f p=q f$. For this, define $q$ so that $q(x)=f p f^{-1}(x)$ if $x \in \operatorname{im}(f)$ and $q(x)=x$ otherwise. Then $q$ is a permutation of $X$ that maps $\operatorname{im}(f)$ onto itself with $S(q) \subseteq f(S(p))$, so that $\operatorname{shift}(q) \leq \operatorname{shift}(p)<\alpha$.

Now if $g=h f^{k}$ for an $h \in S(X, \alpha)$, then

$$
|\operatorname{im}(g)-\operatorname{im}(f)| \leq|h(\operatorname{im}(f))-\operatorname{im}(f)| \leq \operatorname{shift}(h)<\alpha .
$$

Recall that if $H=S(X, \alpha)$ and $\operatorname{shift}(f)<\alpha$ then $\langle f: H\rangle$ is a $\mathscr{G}_{X}$-normal semigroup (Lemma 2.10). Such semigroups are left simple when $\operatorname{def}(f)$ is infinite. So we assume that $\operatorname{shift}(f) \geq \alpha$.

OBSERVATION 3.2. If $\operatorname{def}(f)=\gamma$ is a positive integer, the semigroup $\langle f: H\rangle$ is not simple, so it is not left simple. Indeed, in this case $\langle f: H\rangle$ contains transformations with distinct finite defects (Lemma 2.5). For each positive integer $k \geq 2$ the set $\{g \in\langle f: H\rangle: \operatorname{def}(g) \geq k \gamma\}$ is a proper ideal of $\langle f: H\rangle$.

LEMMA 3.3. Let $f \in \mathscr{W}_{X}$ with $\operatorname{def}(f)=\gamma \geq \aleph_{o}$, and let $H \unlhd \mathscr{G}_{X}$. If $\langle f: H\rangle$ is left simple then $H=S(X, \alpha)$ for some cardinal $\alpha$ with $\gamma<\alpha \leq|X|^{+}$.

Proof. Since $\langle f: H\rangle$ is left simple, there exists $g \in\langle f: H\rangle$ such that $g f^{2}=f$. In this case $g(X-\operatorname{im}(f)) \subseteq X-\operatorname{im}(f)$, so $X-\operatorname{im}(f) \subseteq \operatorname{im}(g)$. By Lemma 3.1, $\alpha>|\operatorname{im}(g)-\operatorname{im}(f)| \geq \gamma$.

COROLlaRY 3.4. If $\operatorname{def}(f)=|X|$ and $H \unlhd \mathscr{G}_{X}$, then $\langle f: H\rangle$ is left simple if and only if $H=\mathscr{G}_{X}$.

LEMMA 3.5. Let $f$ be a non-permutation in $\mathscr{W}_{X}$ with $\operatorname{def}(f)=\gamma<\alpha \leq \operatorname{shift}(f)$. Suppose that $\langle f: S(X, \alpha)\rangle$ is left simple. Then

(1) there exists $g \in\langle f: S(X, \alpha)\rangle$ with $(g)=\gamma$;

(2) there exists a positive integer $k$ such that $\operatorname{shift}\left(f^{k}\right)<\alpha$.

PROOF. To prove (1) take a non-identity permutation $h \in S\left(X, \aleph_{o}\right) \subseteq S(X, \alpha)$ such that $S(h) \subseteq S\left(f_{c}\right)$, where $f_{c}$ is the product of the chains in $f$. Then $D\left(f, h f h^{-1}\right)$ is a 
finite nonempty set. Since $\langle f: S(X, \alpha)\rangle$ is left simple there exists $g \in\langle f: S(X, \alpha)\rangle$ such that $g f=h f h^{-1}$. By Lemma $2.6 \operatorname{shift}(g) \leq \gamma$. Also $g \in\langle f: S(X, \alpha)\rangle$ implies that $\operatorname{def}(g)=\gamma$, so $\operatorname{shift}(g) \geq \gamma$, and hence $\operatorname{shift}(g)=\gamma$.

To prove (2), take $g \in\langle f: S(X, \alpha)\rangle$ with shift $(g)=\gamma$, as constructed above, and write, using Lemma 3.1, $g=h f^{k}$ for some $h \in S(X, \alpha)$, so $\gamma=\operatorname{shift}(g)=$ $\operatorname{shift}\left(h f^{k}\right)=\max \left(\operatorname{shift}(h), \operatorname{shift}\left(f^{k}\right)\right)$. Since $\operatorname{shift}(h)<\alpha$, and $\gamma<\alpha$ we have that $\operatorname{shift}\left(f^{k}\right)<\alpha$.

NOTATION 3.6. Let $f \in \mathscr{W}_{X}$ be such that $\aleph_{o} \leq \operatorname{def}(f)<\alpha \leq \operatorname{shift}(f)$ and $\operatorname{shift}\left(f^{k}\right)<\alpha$ for some positive integer $k$. Use $m$ to denote

$$
m=\min \left\{k \geq 2: k \text { is an integer, } f^{k} \in S(X, \alpha, \gamma)\right\} .
$$

We will use the permutation as constructed below.

NOTATION 3.7. Given a set $A$ of disjoint $n$-cycles, $n=2,3, \ldots ; \infty$, with $|A|=$ $\eta \geq \aleph_{o}$, let $\pi_{A}$ be a permutation of $X$ defined as follows. Choose a subset $B$ of $A$ with $|B|=|A-B|=\eta$ together with a bijection $\lambda$ from $B$ onto $A-B$. For each cycle $u$ in $A$ choose a point $x_{u}$ in $S(u)$ and let $h_{u}$ be the two-cycle $\left(x_{u}, x_{\lambda(u)}\right)$. Let $\pi_{A}=\prod\left\{h_{u}: u \in B\right\}$, and note that $\operatorname{shift}\left(\pi_{A}\right)=\eta$.

If $\langle f: S(X, \alpha)\rangle$ is left simple, then, as the next lemma shows, it contains the semigroup $S(X, \alpha, \gamma)$.

LEMMA 3.8. Suppose that $f \in \mathscr{W}_{X}$ such that $\aleph_{o} \leq \operatorname{def}(f)<\alpha \leq \operatorname{shift}(f)$.

(1) For any cardinal $\eta$ with $\operatorname{def}(f) \leq \eta<\alpha$, there exists a $g \in\langle f: S(X, \alpha)\rangle$ such that $|D(f, g)|=\eta$.

(2) If $\langle f: S(X, \alpha)\rangle$ is left simple, then

(a) $S(X, \alpha, \gamma) \subseteq\langle f: S(X, \alpha)\rangle$,

(b) if $t \in\langle f: S(X, \alpha)\rangle$ and $s \in S\left(X,|X|^{+}, \gamma\right)$ with $|D(s, t)|<\alpha$, then $s \in\langle f: S(X, \alpha)\rangle$,

(c) $\langle f: S(X, \alpha)\rangle=\langle S(X, \alpha), f\rangle$.

PROOF. Let $\operatorname{def}(f)=\gamma, \operatorname{shift}(f)=\sigma$. To prove statement (1) above, write $f=f_{p} f_{c}$ as a product of disjoint transformations $f_{p} \in \mathscr{G}_{X}$ and $f_{c} \in \mathscr{C} h_{X}$. Since $\operatorname{shift}\left(f_{c}\right)=\gamma<\alpha \leq \sigma=\operatorname{shift}(f)$, we have that $\operatorname{shift}\left(f_{p}\right)=\sigma$. So there exists an $n \geq 2$ (finite or infinite) such that $\left|\varphi_{n}(f)\right|=\sigma$. Choose a subset $A$ of $\varphi_{n}(f)$ with $|A|=\eta$ and let $h=\pi_{A}$ (Notation 3.7), $g=h f h^{-1}$. To show that $g$ is the required transformation, it suffices to see that

$$
\eta=|S(h)| \leq|D(f, g)| \leq\left|S\left(\prod\{u: u \in A\}\right)\right| \leq \aleph_{\diamond} \eta=\eta .
$$


For this, take $x \in S(h)$, let $h^{-1}(x)=y \neq x$, and suppose $\left(x x_{1} \cdots\right),\left(y y_{1} \cdots\right)$ are the corresponding $n$-cycles in $A$. Then $g(x)=h f h^{-1}(x)=h f(y)=h\left(y_{1}\right)=y_{1}=$ $f(y) \neq f(x)$, so $S(h) \subseteq D(f, g)$. Now, if $x \in X-S\left(\prod\{u: u \in A\}\right)$, then also $f(x) \in X-S\left(\prod\{u: u \in A\}\right)$, so $g(x)=h f h^{-1}(x)=h f(x)=f(x)$, thus $D(f, g) \subseteq S\left(\prod\{u: u \in A\}\right)$, and so (3.1) follows.

To prove statement (2)(a) of the lemma, we first show that for any $\eta$ with $\gamma \leq \eta<\alpha$ there exists $t \in\langle f: S(X, \alpha)\rangle$ such that $\operatorname{shift}(t)=\eta$. Take $g \in\langle f: S(X, \alpha)\rangle$ with $|D(f, g)|=\eta$ as constructed in the first part of the lemma. Since $\langle f: S(X, \alpha)\rangle$ is left simple, there exists $t \in\langle f: S(X, \alpha)\rangle$ such that $t f=g$. But then by Lemma 2.6 $\operatorname{shift}(t)=\eta$, and the semigroup $\langle f: S(X, \alpha)\rangle$ contains $\langle t: S(X, \alpha)\rangle=S\left(X, \eta^{+}, \gamma\right)$ (Proposition 2.9).

To prove (2) (b) note that since $s, t \in\left\langle f: \mathscr{G}_{X}\right\rangle$, a left simple semigroup, there exists $u \in\left\langle f: \mathscr{G}_{X}\right\rangle$ such that $s=u t$. Since $\operatorname{shift}(u)<\alpha($ Lemma 2.6$), u \in S(X, \alpha, \gamma) \subseteq$ $\langle f: S(X, \alpha)\rangle$, so $s \in\langle f: S(X, \alpha)\rangle$ also. Now (2) (c) follows from (2) (b) and an observation that for any $h \in S(X, \alpha)$, we have that $|D(h f, f)|,|D(f h, f)|<\alpha$.

The next result presents sufficient conditions for the left simplicity of $\langle f: S(X, \alpha)\rangle$.

LEMMA 3.9. Let $f \in \mathscr{W}_{X}$ with $\aleph_{o} \leq \operatorname{def}(f)<\alpha \leq \operatorname{shift}(f)$. Suppose that $\operatorname{shift}\left(f^{m}\right)=\eta<\alpha$ for an integer $m \geq 2$. Then

(1) for any $g \in\langle f: S(X, \alpha)\rangle$ there exists $t \in\langle f: S(X, \alpha)\rangle$ with $\operatorname{shift}(t g) \leq \eta$,

(2) if $v, w \in\langle f: S(X, \alpha)\rangle$ and $\operatorname{shift}(w)=\eta$, then $|D(v w, v)| \leq \eta$,

(3) $\langle f: S(X, \alpha)\rangle$ is left simple.

Proof. To prove (1), write $g=h_{1} f h_{1}{ }^{-1} h_{2} f h_{2}{ }^{-1} \cdots h_{k} f h_{k}^{-1}$ for some $h_{i} \in$ $S(X, \alpha), i=1,2, \ldots, k$. We prove the result by induction on $k$. If $k=1$, $g=h_{1} f h_{1}^{-1}$ and $\operatorname{shift}\left(g^{m}\right)=\operatorname{shift}\left(h_{1} f^{m} h_{1}^{-1}\right)=\operatorname{shift}\left(f^{m}\right)=\eta$, since $S\left(g^{m}\right)=$ $h_{1}\left(S\left(f^{m}\right)\right)$. In this case we let $t=g^{m-1}$.

Assume the result is true for any product of at most $k-1$ conjugates of $f$, where $k \geq 2$, and write $g=u h_{k} f h_{k}{ }^{-1}$, where $u=h_{1} f h_{1}{ }^{-1} h_{2} f h_{2}{ }^{-1} \cdots h_{k-1} f h_{k-1}{ }^{-1}$. By inductive assumption there exists $w \in\langle f: S(X, \alpha)\rangle$ such that $\operatorname{shift}(w u) \leq \eta$. Let $t=\left(h_{k} f h_{k}^{-1}\right)^{m-1} w$. Then

$$
S(t g)=S\left(h_{k} f^{m-1} h_{k}{ }^{-1} w u h_{k} f h_{k}^{-1}\right) \subseteq S\left(h_{k} f^{m} h_{k}^{-1}\right) \cup h_{k} f^{-1} h_{k}^{-1}(S(w u)),
$$

so $\operatorname{shift}(t g) \leq \operatorname{shift}\left(f^{m}\right)+\operatorname{shift}(w u) \leq \eta$.

To prove (2), if $v, w \in \mathscr{W}_{X}$ then $D(v w, v) \subseteq S(w)$, so $|D(v w, v)| \leq \operatorname{shift}(w)$.

To prove (3) note that, by Lemma 2.10 and Proposition $2.9,\langle f: S(X, \alpha)\rangle$ contains a left simple semigroup $\left\langle f^{m}: S(X, \alpha)\right\rangle=\left\langle f^{m}: \mathscr{G}_{X}\right\rangle=S\left(X, \eta^{+}, \gamma\right)$. We show that for any $s, t \in\langle f: S(X, \alpha)\rangle$ there exists $u \in\langle f: S(X, \alpha)\rangle$ such that $u s=t$. Assume first that $|D(s, t)| \leq \eta$. Since $\left\langle f: \mathscr{G}_{X}\right\rangle$ is left simple there exists $u \in\left\langle f: \mathscr{G}_{X}\right\rangle$ such that $u s=t$. By Lemma $2.6 \operatorname{shift}(u) \leq \eta$, and hence $u \in\left\langle f^{m}: \mathscr{G}_{X}\right\rangle \subseteq\langle f: S(X, \alpha)\rangle$. 
Now suppose $|D(s, t)|>\eta$. By statement (1) of this lemma, there exists $w \in\langle f$ : $S(X, \alpha)\rangle$ such that $\operatorname{shift}(w s) \leq \eta$, and so, by statement (2), $|D(t w s, t)| \leq \eta$. Therefore by the previous paragraph there is a $u \in\langle f: S(X, \alpha)\rangle$ such that $u t w s=t$.

ProOF OF THEOREM 2.2. Assume that $\langle f: H\rangle$ is left simple. By Observation 3.2, $\operatorname{def}(f)$ is an infinite cardinal $\gamma$. By Lemma 3.3, the group $H=S(X, \alpha)$ for some $\alpha>\gamma$. By Lemma 3.5 there exists a positive integer $m$ such that $\operatorname{shift}\left(f^{m}\right)<\alpha$. Conversely, if conditions (1)-(3) of Theorem 2.2 hold, then by Lemma 3.9, we have that $\langle f: S(X, \alpha)\rangle$ is a left simple semigroup.

We describe the cyclic-chain structure of $f$ in $\mathscr{W}_{X}$ satisfying the conditions of Lemma 3.9. Let $\operatorname{def}(f)=\gamma \geq \aleph_{o}$, and let $\alpha, \eta$ be cardinals with $\gamma \leq \eta<\alpha \leq|X|^{+}$. Set $\mathscr{N}_{f}^{\eta}=\left\{n:\left|\varphi_{n}(f)\right|>\eta, n\right.$ is an integer, $\left.n \geq 2\right\}$. Note that if the set $\mathscr{N}_{f}^{\eta} \neq \emptyset$, the least common multiple $\operatorname{lcm}\left(\mathscr{N}_{f}^{\eta}\right)$ of the integers in $\mathscr{N}_{f}^{\eta}$ may or may not exist, and set

$$
\mu_{f}^{\eta}= \begin{cases}0 & \text { if } \mathscr{N}_{f}^{\eta}=\emptyset \\ \operatorname{lcm}\left(\mathscr{N}_{f}^{\eta}\right) & \text { if } \operatorname{lcm}\left(\mathscr{N}_{f}^{\eta}\right) \text { is a positive integer, } \\ -1 & \text { otherwise. }\end{cases}
$$

LEMMA 3.10. Let $f$ be in $\mathscr{W}_{X}$ with $\gamma=\operatorname{def}(f) \geq \aleph_{o}$, and let $\eta$ be such that $\gamma \leq \eta<\operatorname{shift}(f)$. The following are equivalent:

(1) There exists a positive integer $m$ such that $\operatorname{shift}\left(f^{m}\right) \leq \eta$;

(2) $\left|\varphi_{\infty}(f)\right| \leq \eta$ and $\mu_{f}^{\eta} \geq 0$.

PROOF. Let $s=\prod\left\{u: u \in \varphi_{\infty}(f)\right\}$, if $\varphi_{\infty}(f)$ is nonempty, and let $s$ be the identity permutation otherwise. Similarly, let $t=\prod\left\{u: u \in \varphi_{n}(f), n\right.$ is an integer, $\left.n \geq 2\right\}$, if $\varphi_{n}(f)$ is nonempty for some integer $n \geq 2$, and let $t$ be the identity permutation otherwise. Then $f=s t f_{c}$, and since $s, t$ and $f_{c}$ are disjoint, for any positive integer $m$ we have that $f^{m}=s^{m} t^{m} f_{c}^{m}$ with $S\left(f^{m}\right)$ being the union of three disjoint subsets: $S\left(s^{m}\right), S\left(t^{m}\right)$ and $S\left(f_{c}^{m}\right)$. Also $S\left(s^{m}\right)=S(s), S\left(t^{m}\right) \subseteq S(t), S\left(f_{c}^{m}\right)=S\left(f_{c}\right)$ ([3]). Since $\operatorname{shift}\left(f_{c}\right)=\operatorname{def}(f) \aleph_{o}=\operatorname{def}(f)$, we have that $\operatorname{shift}\left(f^{m}\right) \leq \eta$ if and only if $\operatorname{shift}\left(s^{m}\right)=\operatorname{shift}(s) \leq \eta$, and $\operatorname{shift}\left(t^{m}\right) \leq \eta$. But $\operatorname{shift}(s)=\left|\varphi_{\infty}\right| \aleph_{o}$, so shift $(s) \leq \eta$ if and only if $\left|\varphi_{\infty}(f)\right| \leq \eta$, and shift $\left(t^{m}\right) \leq \eta$ if and only if $\mathscr{N}_{f}^{\eta}=\emptyset$ or $n$ divides $m$ for all $n \in \mathscr{N}_{f}^{\eta}$, that is $\mu_{f}^{\eta} \geq 0$. Hence (1) and (2) are equivalent.

3.2. Congruences on left simple group closures Given any subsemigroup $S$ of $\mathscr{W}_{X}$ and an infinite cardinal $\mu$, the relation $\Delta_{\mu}=\{(s, t) \in S \times S:|D(s, t)|<\mu\}$ is a congruence on $S$. Let $\Delta$ denote the diagonal congruence on $S$.

Suppose $S$ is the Baer-Levi semigroup $B L(|X|, \gamma)=S\left(X,|X|^{+}, \gamma\right)$ for some infinite cardinal $\gamma$. It was shown in [11] that the interval $\left[\Delta, \Delta_{\gamma^{+}}\right]$is a chain in the congruence lattice $\operatorname{Con}(S)$ of $S$ consisting of $\Delta$ and the set of congruences of 
the form $\Delta_{\eta}$ where $\aleph_{o} \leq \eta \leq \gamma^{+}$with $\Delta_{\eta_{1}} \leq \Delta_{\eta_{2}}$ if and only if $\eta_{1} \leq \eta_{2}$. It was noted in [10] that the arguments of [11], based in part on the left cancellativity and left simplicity of $B L(|X|, \gamma)$ may be adopted virtually unchanged to show that the interval $\left[\Delta, \Delta_{\gamma^{+}}\right]$is a chain in the congruence lattice of any $\mathscr{G}_{X}$-normal subsemigroup of $B L(|X|, \gamma)$. In [10] this result was extended further as follows.

PROPOSITION 3.11. Let $\aleph_{o} \leq \gamma<\sigma$. Then $\operatorname{Con}(S(X, \sigma, \gamma))$ is a chain consisting of $\Delta$ and the set $\left\{\Delta_{\eta}: \aleph_{o} \leq \eta \leq \sigma\right\}$. Moreover, $\Delta_{\gamma^{+}}$is a minimal group congruence on $S(X, \sigma, \gamma)$.

Now let $\langle f: S(X, \alpha)\rangle$ be a left simple semigroup. In view of the above proposition we may assume that it is not a $\mathscr{G}_{X}$-normal semigroup, so (in light of Theorem 2.2 and Lemma 2.10) $\aleph_{o} \leq \operatorname{def}(f)<\alpha \leq \sigma=\operatorname{shift}(f)$, and $\operatorname{shift}\left(f^{m}\right)<\alpha$ for the least positive integer $m \geq 2$. Certain congruences on $\langle f: S(X, \alpha)\rangle$ may be described in terms of congruences on the group $G$ defined below. Note that $\operatorname{shift}\left(f_{p}\right)=\sigma \geq \alpha$, so $f_{p} \notin S(X, \alpha)$, and let

$$
G=\left\langle f_{p}, S(X, \alpha)\right\rangle
$$

be the subgroup of $\mathscr{G}_{X}$ generated by $S(X, \alpha)$ and $f_{p}$. Then

$$
G=\left\{h f_{p}^{k}: h \in S(X, \alpha), k=0,1,2, \ldots, m-1\right\},
$$

and because of the minimality of $m$ this representation of elements of $G$ is unique. Moreover

$$
h f_{p}^{k} \in S(X, \alpha) \Leftrightarrow k \equiv 0 \bmod m \quad \text { and } \quad\left|D\left(h f_{p}^{k}, q f_{p}^{l}\right)\right|<\alpha \Leftrightarrow k \equiv l \bmod m \text {. }
$$

The next result follows from (3.3)-(3.4), Lemma 3.1 and Lemma 3.8 (2) (c).

LEMMA 3.12. Let $f \in \mathscr{W}_{X}$ be such that $\aleph_{o} \leq \gamma=\operatorname{def}(f)<\alpha \leq \operatorname{shift}(f)$ and $\operatorname{shift}\left(f^{m}\right)<\alpha$. Then $\langle f: S(X, \alpha)\rangle=\left\{h t: h \in G, t \in \mathscr{C} h_{X} \cap S\left(X, \gamma^{+}, \gamma\right), h, t\right.$ are disjoint\}.

OBSERVATION 3.13 (Normal subgroups of $G$ ). Let $H$ be a non-trivial normal subgroup of $G$. If $H \leq S(X, \alpha)$, then $H=S(X, \eta)$, for some infinite cardinal $\eta \leq \alpha$, or $H=\mathscr{A}_{X}$ [12]. Suppose $H$ contains $g=h f_{p}^{k} \notin S(X, \alpha)$. We show that $H$ contains $S(X, \alpha)$. Let $\aleph_{o} \leq \xi<\alpha$. Since $\operatorname{shift}(g) \geq \alpha$, choose a subset $Y$ of $S(g)$ of cardinality $\xi$ such that $g(Y)=Y$. There exists a set $A$ of disjoint $n$-cycles of $g_{\mid Y}$ for some $n \in\{2,3, \ldots\} \cup\{\infty\}$ such that $|A|=\xi$. Form a set $B$ of $n$-cycles of $X$ by extending the cycles of $A$ to $X$ by fixing elements of $X-Y$. Now form $\pi_{B}$ (Notation 3.7) so $\pi_{B} \in S\left(X, \xi^{+}\right)$. Consider the commutator $y=g^{-1} \pi_{B} g \pi_{B}^{-1} \in H$. Then $y$ is a 
product of $2 \xi=\xi$ disjoint 2-cycles, so shift $(y)=\xi^{+}$. Hence $S\left(X, \xi^{+}\right)$, the normal closure of $y$ in $S(X, \alpha)$, is contained in $H$. But $\xi$ is arbitrary, so $S(X, \alpha) \subseteq H$. Since $G / S(X, \alpha) \cong \mathbb{Z}_{m}$, such normal subgroups $H$ correspond to normal subgroups of $\mathbb{Z}_{m}$ : $H=\left\{h f_{p}^{k} \in G: k \in\langle n\rangle \unlhd \mathbb{Z}_{m}\right\}$ where $n$ is a divisor of $m$.

We may easily adapt the arguments of [11] to show that the interval $\left[\Delta, \Delta_{\gamma^{+}}\right]$is a chain in $\operatorname{Con}(\langle f: S(X, \alpha)\rangle)$ consisting of $\Delta$ and the set of congruences of the form $\Delta_{\eta}$ where $\aleph_{o} \leq \eta \leq \gamma^{+}$. The proof may be accomplished using the elements of the $\mathscr{G}_{X}$-normal subsemigroup $S(X, \alpha, \gamma)$ of $\langle f: S(X, \alpha)\rangle$ with the aid of Lemma 3.8 (2). The consequence of this is stated below.

LEMMA 3.14. Let $f \in \mathscr{W}_{X}$ with $\aleph_{o} \leq \gamma=\operatorname{def}(f)<\alpha \leq \sigma=\operatorname{shift}(f)$, and $\operatorname{shift}\left(f^{m}\right)<\alpha$ for some positive integer $m$. Then the interval $\left[\Delta, \Delta_{\gamma^{+}}\right]$is a chain in $\operatorname{Con}(\langle f: S(X, \alpha)\rangle)$ consisting of $\Delta$ and the set $\left\{\Delta_{\eta}: \aleph_{o} \leq \eta \leq \gamma^{+}\right\}$. Moreover, $\Delta_{\gamma^{+}}$is a minimal group congruence on $\langle f: S(X, \alpha)\rangle$, and if $\theta$ is a congruence not in $\left[\Delta, \Delta_{\gamma^{+}}\right]$then $\Delta_{\gamma^{+}} \subseteq \theta$.

For each divisor $n$ of $m, 1 \leq n<m$, define the relation

$$
\Gamma_{n}^{\prime}=\left\{\left(h f_{p}^{k}, q f_{p}^{l}\right): h, q \in S(X, \alpha), k \equiv l \bmod n\right\}
$$

on $G$. Then the relation

$$
\Gamma_{n}=\left\{(s, t): s, t \in\langle f: S(X, \alpha)\rangle,\left(s_{p}, t_{p}\right) \in \Gamma_{n}^{\prime}\right\}
$$

is a congruence on $\langle f: S(X, \alpha)\rangle$ that contains $\Delta_{\alpha}$. The next lemma describes the congruences of $\langle f: S(X, \alpha)\rangle$ that contain $\Delta_{\gamma^{+}}$. Let $\nabla$ denote the universal congruence.

LEMMA 3.15. Let $f \in \mathscr{W}_{X}$ with $\aleph_{o} \leq \gamma=\operatorname{def}(f)<\alpha \leq \operatorname{shift}(f)$, and $\operatorname{shift}\left(f^{m}\right)<\alpha$ for some positive integer $m$.

(1) $\langle f: S(X, \alpha)\rangle / \Delta_{\gamma^{+}} \cong G / \Delta_{\gamma^{+}}$.

(2) The interval $\left[\Delta_{\gamma^{+}}, \Delta_{\alpha}\right]$ in $\operatorname{Con}(\langle f: S(X, \alpha)\rangle)$ is a chain consisting of $\left\{\Delta_{\eta}\right.$ : $\left.\gamma^{+} \leq \eta \leq \alpha\right\}$.

(3) The interval $\left[\Delta_{\alpha}, \nabla\right]$ is a chain consisting of $\nabla$ and $\left[\Gamma_{n}: n\right.$ is a divisor of $m$, $1 \leq n<m\}$.

(4) The interval $\left[\Delta_{\gamma^{+}}, \nabla\right]$ in $\operatorname{Con}(\langle f: S(X, \alpha)\rangle)$ is a chain with $\left[\Delta_{\gamma^{+}}, \nabla\right]=$ $\left[\Delta_{\gamma^{+}}, \Delta_{\alpha}\right] \cup\left[\Delta_{\alpha}, \nabla\right]$.

PROOF. Observe that statements (2) and (3) follow from (1) and Observation 3.13, while statement (4) follows from (2), (3) and the last sentence of the statement of Lemma 3.14. To prove (1), let $g \in\langle f: S(X, \alpha)\rangle$. By Lemma 3.12, $g_{p} \in G$. Let $\bar{g}$ be the class of $g$ in $\langle f: S(X, \alpha)\rangle / \Delta_{\gamma^{+}}$, and let $\overline{g_{p}}$ be the class of $g_{p}$ in $G / \Delta_{\gamma^{+}}$. Let $\psi:\langle f: S(X, \alpha)\rangle / \Delta_{\gamma^{+}} \rightarrow G / \Delta_{\gamma^{+}}$be defined by $\psi: \bar{g} \mapsto \overline{g_{p}}$. 
To show that $\psi$ is well-defined and one-to-one, take $g=g_{p} g_{c}, t=t_{p} t_{c} \in\langle f$ : $S(X, \alpha)\rangle$. Since $\operatorname{shift}\left(g_{c}\right)$, shift $\left(t_{c}\right)=\gamma$, we have that $|D(g, t)| \leq \gamma$ if and only if $\left|D\left(g_{p}, t_{p}\right)\right| \leq \gamma$, so $\bar{t}=\bar{g}$ if and only if $\overline{t_{p}}=\overline{g_{p}}$. To see that $\psi$ is onto, take any $h f_{p}^{k} \in G$, then $u=h f_{p}^{k} f_{c}^{k} \in S(X, \alpha)\langle f\rangle=\langle f: S(X, \alpha)\rangle$ (Lemma 3.8). Let $u=u_{p} u_{c}$. Since $\operatorname{shift}\left(f_{c}^{k}\right), \operatorname{shift}\left(u_{c}\right)=\gamma$, we have that $\left|D\left(h f_{p}^{k}, u_{p}\right)\right| \leq \gamma$, so $\overline{h f_{p}^{k}}=\overline{u_{p}}=\psi(\bar{u})$.

To see that $\psi$ is a homomorphism, take $g=g_{p} g_{c}, t=t_{p} t_{c} \in\langle f: S(X, \alpha)\rangle$, and let $g t=u=u_{p} u_{c}$. Again since the shift of the $\gamma$ chains $g_{c}, t_{c}, u_{c}$ is $\gamma$, and $u_{p} u_{c}=u=g t=g_{p} t_{p}\left(t_{p}^{-1} g_{c} t_{p}\right) t_{c}$, we have that

$$
\begin{aligned}
\left|D\left(u_{p}, g_{p} t_{p}\right)\right| & \leq\left|D\left(u_{p}, u_{p} u_{c}\right)\right|+\left|D\left(u_{p} u_{c}, g_{p} t_{p}\right)\right| \leq \gamma+S\left(t_{p}^{-1} g_{c} t_{p} t_{c}\right) \mid \\
& \leq \gamma+\left|S\left(t_{p}^{-1} g_{c} t_{p}\right)\right|+\left|S\left(t_{c}\right)\right| \leq \gamma+\gamma+\gamma=\gamma,
\end{aligned}
$$

and so $\psi(\bar{u})=\overline{u_{p}}=\overline{g_{p}} \bar{t}_{p}=\psi(\bar{g}) \psi(\bar{t})$.

3.3. Automorphisms Recall that given any semigroup $S$ of transformations of $X$, $G_{S}=\left\{h \in \mathscr{G}_{X}: h S h^{-1} \subseteq S\right\}$. An automorphism $\vartheta$ of $S$ is said to be inner if there exists a permutation $h$ of $X$ such that $\vartheta: f \mapsto h f h^{-1}$, for each $f \in S$. A description of automorphisms of $\langle f: H\rangle$, presented below, may be found in [5].

PROPOSITION 3.16. Let $f$ be a non-permutation in $\mathscr{W}_{X}$, and let $H$ be a non-identity normal subgroup of $\mathscr{G}_{X}$. Then each automorphism of $\langle f: H\rangle$ is inner, and the automorphism group of $\langle f: H\rangle$ is isomorphic to $G_{(f: H\rangle}$.

PROOF OF THEOREM 2.3. The non-trivial congruences of a left simple semigroup $S=\langle f: S(X, \alpha)\rangle$ have the form $\Delta_{\eta}=\{(s, t): s, t \in\langle f: S(X, \alpha)\rangle,|D(s, t)|<\eta\}$ for $\aleph_{o} \leq \eta \leq \alpha$, or

$$
\begin{gathered}
\Gamma_{n}=\left\{(s, t): s, t \in\langle f: S(X, \alpha)\rangle, s_{p}=h f_{p}^{k}, t_{p}=q f_{p}^{l}, h, q \in S(X, \alpha),\right. \\
k \equiv l \bmod n\},
\end{gathered}
$$

for a divisor $n$ of $m, 1 \leq n<m$ (Lemma 3.14, Lemma 3.15). Since for any permutation $r \in \mathscr{G}_{X}$, and any transformations $s, t$ of $X,\left|D\left(h s h^{-1}, h t h^{-1}\right)\right|=|D(s, t)|$, and all the automorphisms of $\langle f: S(X, \alpha)\rangle$ are inner, its congruences of the form $\Delta_{\mu}$ are preserved under automorphisms. Similarly, conjugation by permutations preserves the congruences $\Gamma_{n}$.

\section{Equality of group closures}

We start by considering $H$-closures of $f \in \mathscr{W}_{x}$ with $H=S(X, \alpha)$. In view of Lemma 2.10, we may concentrate on transformations with a 'large' shift, that is $\operatorname{shift}(f) \geq \alpha$. 
LEMMA 4.1. Let $\aleph_{o} \leq \alpha<\beta \leq|X|^{+}$and take $f \in \mathscr{W}_{X}$ with a finite non-zero defect and $\operatorname{shift}(f) \geq \alpha$. Then $\langle f: S(X, \alpha)\rangle \neq\langle f: S(X, \beta)\rangle$.

ProOF. To show that $\langle f: S(X, \alpha)\rangle$ and $\langle f: S(X, \beta)\rangle$ are distinct, it suffices to find an $h$ in $S(X, \beta)$ such that $h f h^{-1} \notin\langle f: S(X, \alpha)\rangle$. Since $\operatorname{def}(f)$ is finite, $h f h^{-1} \in\langle f: S(X, \alpha)\rangle$ if and only if $h f h^{-1}=q f q^{-1}$ for some $q \in S(X, \alpha)$ (Lemma 2.5 (1)), that is $q^{-1} h \in C_{\mathscr{G}_{X}}(f)$. We will find an $h \in S(X, \beta)$ such that $q^{-1} h \in \mathscr{G}_{X}-C_{\mathscr{G}_{X}}(f)$ for all $q \in S(X, \alpha)$.

Since $\operatorname{def}(f)$ is finite, $\operatorname{shift}\left(f_{c}\right)=\operatorname{def}(f) \aleph_{o}=\aleph_{o}$. Suppose first that $\operatorname{shift}(f)=$ $\sigma>\aleph_{o}$. Then $\operatorname{shift}\left(f_{p}\right)=\sigma \geq \alpha$, and there exists an $n=2,3,4, \ldots, \infty$ such that $\left|\varphi_{n}(f)\right|=\sigma$. Choose a subset $A$ of $\varphi_{n}(f)$ of cardinality $\alpha$, and let $h=\pi_{A}$ (Notation 3.7), so $\operatorname{shift}(h)=\alpha$ and $h \in S(X, \beta)-S(X, \alpha)$. Suppose that $q \in \mathscr{G}_{X}$ such that $q^{-1} h \in C_{\mathscr{G}_{X}}(f)$. Then $q^{-1} h \in C_{\mathscr{G}_{X}}\left(f_{p}\right)$ and $q^{-1} h$ maps each $n$-cycle of $f_{p}$ onto an $n$-cycle of $f_{p}$ (by Proposition 2.7), and so $\operatorname{shift}\left(q^{-1}\right) \geq \operatorname{shift}(h)=\alpha$. Therefore $q^{-1} \notin S(X, \alpha)$.

Now suppose that $\operatorname{shift}(f)=\aleph_{o}$, so $S(X, \alpha)=S\left(X, \aleph_{o}\right)$. Take a chain $\left[x_{1} x_{2} \cdots\right)$ in $f_{c}$ and let $h$ be the product of disjoint transpositions, $h=\left(x_{1} x_{2}\right)\left(x_{3} x_{4}\right) \cdots$. The permutation $h$ shifts a countable number of points of $X$, so $h \in S(X, \beta)-S(X, \alpha)$. It follows from Proposition 2.7 that if $q^{-1} h \in C_{\mathscr{G}_{X}}(f)$ then $\left[q^{-1} h\left(x_{1}\right) q^{-1} h\left(x_{2}\right) \cdots\right)$ is a chain in $f_{c}$, so shift $\left(q^{-1}\right) \geq \aleph_{o}$, and again $q^{-1} \notin\langle f: S(X, \alpha)\rangle$.

Our aim now is to extend the above result to a transformation $f$ with an infinite or zero defect.

LEMMA 4.2. Let $\aleph_{0} \leq \alpha<\beta \leq|X|^{+}$, and let $f \in \mathscr{W}_{X}$ with $\operatorname{def}(f) \geq \alpha$. Then $\langle f: S(X, \alpha)\rangle \neq\langle f: S(X, \beta)\rangle$.

Proof. Choose a subset $A$ of $\operatorname{im}(f)$ with $|A|=\alpha$, and a permutation $h \in S(X, \beta)$ such that $h(A) \subseteq X-\operatorname{im}(f)$. Set $g=h f h^{-1} \in\langle f: S(X, \beta)\rangle$. Then $\operatorname{im}(g)=$ $h(\operatorname{im}(f))$, and $|\operatorname{im}(g)-\operatorname{im}(f)| \geq \alpha$, so $g \notin\langle f: S(X, \alpha)\rangle$ by Lemma 3.1.

The next result is concerned with $f \in \mathscr{W}_{X}$ that has both shift and fix at least as large as $\alpha$.

LEMMA 4.3. Let $\aleph_{o} \leq \alpha<\beta \leq|X|^{+}$and let $f \in \mathscr{W}_{X}$ with $\operatorname{shift}(f) \geq \alpha$ and $\operatorname{fix}(f) \geq \alpha$. Then $\langle f: S(X, \alpha)\rangle \neq\langle f: S(X, \beta)\rangle$.

PROOF. For any $t \in\langle f: S(X, \alpha)\rangle$, we have that $|S(t)-S(f)|<\alpha$. Indeed, by Lemma 3.1, $t=h f^{k}$ for some $h \in S(X, \alpha)$ and an integer $k \geq 1$. Then $S(t)-S(f) \subseteq$ $S(h)$, so $|S(t)-S(f)| \leq \operatorname{shift}(h)<\alpha$. 
We construct a transformation $g \in\langle f: S(X, \beta)\rangle$ such that $|S(g)-S(f)| \geq \alpha$. For this choose sets $A \subseteq X-S(f)$ and $B \subseteq S(f)$ of cardinality $\alpha$ each. Choose a bijection $r$ from $A$ onto $B$ and define a permutation $h$ of $x$ as follows:

$$
h(x)= \begin{cases}r(x) & \text { if } x \in A \\ r^{-1}(x) & \text { if } x \in B \\ x & \text { otherwise }\end{cases}
$$

Then shift $(h)=|A \cup B|=\alpha<\beta$, so $h \in S(X, \beta)$, and $g=h f h^{-1} \in\langle f: S(X, \beta)\rangle$ is the required transformation, since $A \subseteq S(g)-S(f)$.

LEMMA 4.4. Let $\aleph_{o}<\alpha<\beta \leq|X|^{+}, f \in \mathscr{W}_{X}$ with $\operatorname{def}(f)<\alpha$, and $\operatorname{fix}(f)<\alpha$. Then $\langle f: S(X, \alpha)\rangle \neq\langle f: S(X, \beta)\rangle$.

ProOF. Observe that the sum of $\operatorname{shift}(f)$ and $\operatorname{fix}(f)$ is $|X|$. Since $\operatorname{fix}(f)$ is less than $\alpha$ we have that $\operatorname{shift}(f)=|X|$. Since $\operatorname{shift}\left(f_{c}\right)=\operatorname{def}(f) \aleph_{o}<\alpha \aleph_{o}=\alpha$, we have that $\operatorname{shift}\left(f_{p}\right)=|X|$. Thus there exists a largest $n \geq 2$ (finite or infinite) such that $\left|\varphi_{n}(f)\right|=|X|$. Let $C=S\left(\prod\left\{c: c \in \varphi_{n}(f)\right\}\right)$, then $|C|=n|X|=|X|$, since $n$ is countable. We consider the following three cases.

Case 1. $|X-C| \geq \alpha$. For any $t \in\langle f: S(X, \alpha)\rangle$, we have that $|t(C)-C|<\alpha$. Indeed, using Lemma 3.1, write $t=h f^{k}$ for some $h \in S(X, \alpha)$ and an integer $k \geq 1$. Then $|t(C)-C|=\left|h f^{k}(C)-C\right|=|h(C)-C| \leq \operatorname{shift}(h)<\alpha$. We show that there exists $g \in\langle f: S(X, \beta)\rangle$ such that $|g(C)-C| \geq \alpha$. Choose a subset $B$ of $\varphi_{n}(f)$ of cardinality $\alpha$. For each cycle $c$ in $B$ choose a point in $S(c)$ and let $K$ be the set of all the chosen points. Set $N=S\left(\prod\{c: c \in B\}\right)-K$. Since $n \geq 2$ and $\alpha$ is infinite, we have that $|K|=|N|=\alpha$. The shift of $f_{c}$ is $\aleph_{o} \operatorname{def}(f)<\alpha$. Since $|X-C| \geq \alpha$, we may choose a subset $L$ of $X-C-S\left(f_{c}\right)$ of cardinality $\alpha$. Choose bijections $r_{1}$ from $K$ onto $N, r_{2}$ from $N$ onto $L$, and $r_{3}$ from $L$ onto $K$. Take a permutation $h \in S(X, \beta)$ defined as follows:

$$
h(x)= \begin{cases}r_{1}(x) & \text { if } x \in K \\ r_{2}(x) & \text { if } x \in N \\ r_{3}(x) & \text { if } x \in L \\ x & \text { otherwise }\end{cases}
$$

Then $g=h f h^{-1}$ is the required transformation. Indeed, if $x \in N \subseteq C$, then $h^{-1}(x) \in K$, and since $n \geq 2$ we have $f\left(h^{-1}(x)\right) \in N$. Therefore,

$$
g(N)=h f h^{-1}(N) \subseteq h(f(K)) \subseteq h(N) \subseteq L \subseteq X-C,
$$

so $g(C)-C$ contains the set $g(N)$ of cardinality $\alpha$, as required. 
Case 2. $|X-C|<\alpha, n$ is an integer. In this case $\operatorname{shift}\left(f^{n}\right) \leq|X-C|<\alpha<\beta$, so by Theorem $2.2,\langle f: S(X, \alpha)\rangle$ and $\langle f: S(X, \beta)\rangle$ are left simple semigroups. Therefore $\langle f: S(X, \beta)\rangle$ contains $S(X, \beta, \gamma)$, where $\gamma=\operatorname{def}(f)$ (Lemma 3.8). Take a set $A$ of $\alpha$ disjoint infinite cycles and a set $B$ of $\gamma$ disjoint chains so that the cycles in $A$ and the chains in $B$ are also disjoint. Then $g=\prod\{c: c \in A \cup B\} \in$ $S(X, \beta, \gamma)-\langle f: S(X, \alpha)\rangle$ (Lemma 3.12).

Case 3. $|X-C|<\alpha, n=\infty$. For all $t \in\langle f: S(X, \alpha)\rangle$ we have that fix $(t)<\alpha$. Indeed write $t=h f^{k}$ for some $h \in S(X, \alpha)$ and an integer $k \geq 1$, and note that $C \subseteq S\left(f^{k}\right)$. Then fix $(t) \leq|S(h) \cup(X-C)|<\alpha$.

We show that there exists $g \in\langle f: S(X, \beta)\rangle$ such that fix $(g) \geq \alpha$. Choose a subset $B$ of $\varphi_{\infty}(f)$ of cardinality $\alpha$, and let $A=S\left(\prod\{c: c \in B\}\right)$. Then $f(A)=A$ and the restriction $f_{\mid A}$ of $f$ to $A$ is a product of disjoint infinite cycles. Therefore, there exists a permutation $r$ of $A$ such that $r f_{\mid A} r^{-1}=f^{-1}$. Define a permutation $h$ of $X$ so that $h(x)=r(x)$ if $x \in A$, and $f(x)=x$ otherwise. Then $\operatorname{shift}(h)=\alpha$, so $h \in$ $S(X, \beta)-S(X, \alpha)$, and $g=h f h^{-1} f \in\langle f: S(X, \beta)\rangle$ is the desired transformation. Indeed $A \subseteq X-S(g)$ and so $\operatorname{fix}(g) \geq \alpha$.

PROOF OF THEOREM 2.4. We first prove, under the assumption $\alpha<\beta$, that $\langle f$ : $S(X, \alpha)\rangle=\langle f: S(X, \beta)\rangle$ if and only if $\operatorname{shift}(f)<\alpha$. Indeed, if $\operatorname{shift}(f)<\alpha$, then Lemma 2.10 ensures that $\langle f: S(X, \alpha)\rangle=\left\langle f: \mathscr{G}_{X}\right\rangle=\langle f: S(X, \beta)\rangle$. Suppose that $\operatorname{shift}(f) \geq \alpha$, we prove that $\langle f: S(X, \alpha)\rangle \neq\langle f: S(X, \beta)\rangle$. Lemmas 4.1 and 4.2 prove the result if $\operatorname{def}(f)$ is either a positive integer or an infinite cardinal greater than or equal to $\alpha$. If $\operatorname{fix}(f) \geq \alpha$, the result is proven in Lemma 4.3, while if $\operatorname{fix}(f)<\alpha$ and $\operatorname{def}(f)<\alpha$, the result is proven in Lemma 4.4.

We now turn to the second part of Theorem 2.4, establishing the conditions for equality

$$
\left\langle f: \mathscr{A}_{X}\right\rangle=\langle f: S(X, \beta)\rangle .
$$

Since $\left\langle f: \mathscr{A}_{X}\right\rangle \subseteq\left\langle f: S\left(X, \aleph_{a}\right)\right\rangle \subseteq\langle f: S(X, \beta)\rangle,(4.1)$ is equivalent to

$$
\left\langle f: \mathscr{A}_{X}\right\rangle=\left\langle f: S\left(X, \aleph_{o}\right)\right\rangle \text { and }\left\langle f: S\left(X, \aleph_{o}\right)\right\rangle=\langle f: S(X, \beta)\rangle .
$$

If $f \in \mathscr{A}_{X}$, then $\left\langle f: \mathscr{A}_{X}\right\rangle$ is a normal subgroup of $\mathscr{A}_{X}$, so $\left\langle f: \mathscr{A}_{X}\right\rangle=\mathscr{A}_{X}=$ $\left\langle f: S\left(X, \aleph_{o}\right)\right\rangle=\left\langle f: \mathscr{G}_{X}\right\rangle$. If $f \in S\left(X, \aleph_{o}\right)-\mathscr{A}_{X}$ then $\left\langle f: \mathscr{A}_{X}\right\rangle \supset \mathscr{A}_{X}$, and so $\left\langle f: \mathscr{A}_{X}\right\rangle=S\left(X, \aleph_{o}\right)=\left\langle f: S\left(X, \aleph_{o}\right)\right\rangle=\left\langle f: \mathscr{G}_{X}\right\rangle$.

We assume now that (4.1) holds and $f$ is a non-permutation, so $\operatorname{shift}(f) \geq \aleph_{o}$. Then it follows from the second equation of (4.2) and the first part of Theorem 2.4 that $\beta=\aleph_{o}$. Let $q$ be a finite odd permutation, and let $t=q f q^{-1} \in\left\langle f: S\left(X, \aleph_{o}\right)\right\rangle$ $=\left\langle f: \mathscr{A}_{X}\right\rangle$. We will show that $t=h f h^{-1}$ for some $h \in \mathscr{A}_{X}$. Indeed, there 
exist permutations $h_{1}, \ldots, h_{k} \in \mathscr{A}_{X}$ that $t=h_{1} f h_{1}{ }^{-1} h_{2} f h_{2}{ }^{-1} \ldots h_{k} f h_{k}{ }^{-1}$. By Lemma 3.1, there exists $q \in S\left(X, \aleph_{o}\right)$ such that $t=q f^{k}$. If $\operatorname{def}(f)$ is finite, then $k=1$ by Lemma $2.5(1)$. Assume that $\operatorname{def}(f)$ is infinite. Since $\operatorname{shift}(q)$ is finite, $|\operatorname{im}(f)-\operatorname{im}(t)|=|\operatorname{im}(f)-q(\operatorname{im}(f))|<\aleph_{o}$. If $k \geq 2,\left|\operatorname{im}(f)-\operatorname{im}\left(f^{k}\right)\right| \geq$ $\left|\operatorname{im}(f)-\operatorname{im}\left(f^{2}\right)\right|=\operatorname{def}(f) \geq \aleph_{o}, \operatorname{so}|\operatorname{im}(f)-\operatorname{im}(t)| \geq \aleph_{o}$ also. Therefore, $k=1$ again. Thus $t=h f h^{-1}$ for some $h \in \mathscr{A}_{X}$.

The equality $q f q^{-1}=h f h^{-1}$ implies that the odd permutation $q^{-1} h$ is in $C_{\mathscr{G}_{X}}(f)$. Hence $C_{\mathscr{G}_{X}}(f) \cap S\left(X, \aleph_{o}\right) \nsubseteq \mathscr{A}_{X}$. Let $g$ be an identity transformation of $X$ if $f_{p}$ has no infinite cycles, and $g=\prod\left\{c: c \in \varphi_{\infty}(f)\right\}$ otherwise. By Proposition 2.7, $q^{-1} h \in C_{G_{X}}\left(f_{p}\right)$ and $q^{-1} h$ is the identity on $S\left(f_{c} g\right)$. Set $Y=X-S\left(f_{c} g\right)$. Then the restriction $q^{-1} h_{\mid Y}$ of $q^{-1} h$ to $Y$ is a finite odd permutation in $C_{G_{Y}}\left(f_{\mid Y}\right)$. Applying Lemma 2.8 to $\left(f_{p}\right)_{\mid Y}$ we deduce that $\left(f_{p}\right)_{\mid Y}$ contains either a $(2 k)$-cycle or at least two ( $2 k-1)$-cycles for some integer $k \geq 1$ (so if $k=1$ then $\left(f_{p}\right)_{\mid Y}$ contains at least two fixed points).

Conversely, assume that $\beta=\aleph_{o}$ and $f$ contains either a $(2 k)$-cycle or at least two $(2 k-1)$-cycles for some integer $k \geq 1$. Since the index $\left[S\left(X, \aleph_{o}\right): \mathscr{A}_{X}\right]=2$, adopting the argument of [4], we deduce that to demonstrate the equality $\left\langle f: \mathscr{A}_{X}\right\rangle=$ $\left\langle f: S\left(X, \aleph_{o}\right)\right\rangle$ we only need to construct $h \in S\left(X, \aleph_{o}\right)-\mathscr{A}_{X}$ such that $h f h^{-1} \in\langle f:$ $\left.\mathscr{A}_{X}\right\rangle$. If $\varphi_{2 k}(f) \neq \emptyset$ for some integer $k \geq 1$, choose $h$ to be a cycle in $\varphi_{2 k}(f)$ and then $h f h^{-1}=f \in\left\langle f: \mathscr{A}_{X}\right\rangle$. If $\left|\varphi_{2 k-1}(f)\right| \geq 2$ for some integer $k \geq 1$, choose two distinct $(2 k-1)$-cycles $\left(x_{1} x_{2} \cdots x_{2 k-1}\right)$ and $\left(y_{1} y_{2} \cdots y_{2 k-1}\right)$ in $\varphi_{2 k-1}(f)$ and let $h=\left(x_{1} y_{1}\right)\left(x_{2} y_{2}\right) \ldots\left(x_{2 k-1} y_{2 k-1}\right)$ be the indicated product of transpositions, so again $h f h^{-1}=f \in<f: \mathscr{A}_{X}>$. Finally, if $f$ is a finite permutation then all conjugates of $f$ are also finite permutations and it follows quickly that $\left\langle f: \mathscr{A}_{X}\right\rangle=\langle f: S(X, \beta)\rangle$, completing the proof of the theorem.

\section{References}

[1] A. H. Clifford and G. B. Preston, The algebraic theory of semigroups, Math. Surveys 7, Vol. I (Amer. Math. Soc., Providence, RI, 1961).

[2] I. Levi, 'Automorphisms of normal transformation semigroups', Proc. Edinburgh Math. Soc. 28 (1985), 185-205.

[3] —_, 'Normal semigroups of one-to-one transformations', Proc. Edinburgh Math. Soc 34 (1991), 65-76.

[4] - 'On the inner automorphisms of finite transformation semigroups', Proc. Edinburgh Math. Soc. 30 (1996), 27-30.

[5] — ' 'On groups associated with transformation semigroups', Semigroup Forum 59 (1999), 342-353.

[6] - 'Group closures of one-to-one transformations', Bull. Austral. Math. Soc. 64 (2001), 177-188. 
[7] I. Levi, D. B. McAlister and R. B. McFadden, 'Groups associated with finite transformation semigroups', Semigroup Forum 61 (2000), 453-467.

[8] —_ ' $\mathscr{A}_{n}$-normal semigroups', Semigroup Forum 62 (2001), 173-177.

[9] I. Levi, B. M. Schein, R. P. Sullivan and G. R. Wood, 'Automorphisms of Baer-Levi semigroups', J. London Math. Soc. 28 (1983), 492-495.

[10] I. Levi and S. Seif, 'On congruences of $\mathscr{G}_{X}$-normal semigroups', Semigroup Forum 43 (1991), 93-113.

[11] D. Lindsey and B. Madison, 'The lattice of congruences on a Baer-Levi semigroup', Semigroup Forum 12 (1976), 63-70.

[12] W. R. Scott, Group theory (Prentice Hall, NJ, 1964).

[13] R. P. Sullivan, 'Automorphisms of transformation semigroups', J. Austral. Math. Soc. 20 (1975), 77-84.

[14] E. G. Sutov, 'On semigroups of almost identical transformations', Soviet Math. Dokl. 1 (1960), 1080-1083.

Department of Mathematics

Morgan Hall 118

1 University Circle

Western Illinois University

Macomb, IL 61455-1390

USA

e-mail: I-Levi@wiu.edu 